\title{
Design on a Desktop Meal-assistance Robot
}

\author{
FKerong Gai ${ }^{1, a}$, Jinxiang Zeng ${ }^{1, b}$ \\ ${ }^{1}$ Beijing polytechnic college, No.368 Shimen Road Shijingshan District,Beijing in China \\ agkr0706@126.com binsam520@126.com
}

Keywords: desktop robot, meal assistance, voice interaction

Abstract. To cater for the demands to desktop meal-assistance robots, the paper designs a meal-assistance bluetooth-controlled robot which applies the wireless, embedded S3C6410 as the processor with ARM11 as the core, realizes voice interaction, foot-switch control and other functions through transplanting Linux-2.6.36 operation system and applying the RTP protocol and Media streamer 2 stream media technology for the transmission of audio/video data, and displays food information through through playing audio/video files on the screen. The structure and work principle are also elaborated in the paper. The field test indicates that the developed prototype robot features simple structure and stable performance and can complete the meal assistance to the senior or disabled persons in old people's home, community or ordinary home environment. This template explains and demonstrates how to prepare your camera-ready paper for Trans Tech Publications. The best is to read these instructions and follow the outline of this text.

\section{Introduction}

With the further development of the social aging trend and the fading of population dividend, the care to the dining of some senior or upper-limb-disabled persons becomes an upcoming problem[1]. In order to help them dine pleasantly, the paper, with reference to other international products [2-6], designs a meal-assistance machine which is easy to control and tailor-made for Chinese dining habit. Based on computing and processing technology, artificial intelligence, remote sensing technology and Internet technology, home service robots integrating functions for aiding and caring for the senior, housework and entertainment will enter homes extensively at a high speed. The paper designs a stable desktop meal-assistance machine that can achieve diverse dining modes, spoon eating assistance, upward and downward adjustment and voice interaction through easy control modes, laying a foundation for further smart development of service robots.

\section{The general design plan}

The desktop meal-assistance machine consists of two parts, the mechanical part which mainly includes container lifting/lowering/rotating and spoon feeding units, and the control system which includes foot-control module, voice interaction module and safety module, The general design plan is illustrated in Figure 1.

The system, with the Controller Samsung ARM11 S3C6410 as the core, runs Linux-2.6.36 kernel operation system ${ }^{[1]}$. UDA1341 is included to drive the voice encoding/decoding equipment, and directly compile the codes into the kernel. The system controls five motors for lifting, rotation, horizontal/vertical threaded spindles and steering engine of the spoon respectively. The system also has a network-based LCD for synchronous menu display

\section{Structure and principle of desktop meal-assistance machine}

The mechanical structure of meal-assistance robot is illustrated in Figure 2. Smart meal-assistance robot consists of two parts. One part is the bowl seat which can be lifted, lowered or rotated and have four U-shaped bowls with the bottom curvature radius same with that of the spoon. The design is helpful to scoop food and reduce remaining food. The U-shaped bowls are symmetrically buckled on the seat, which is easy for disassembly and cleaning. The meal-assistance machine can be adjusted to 


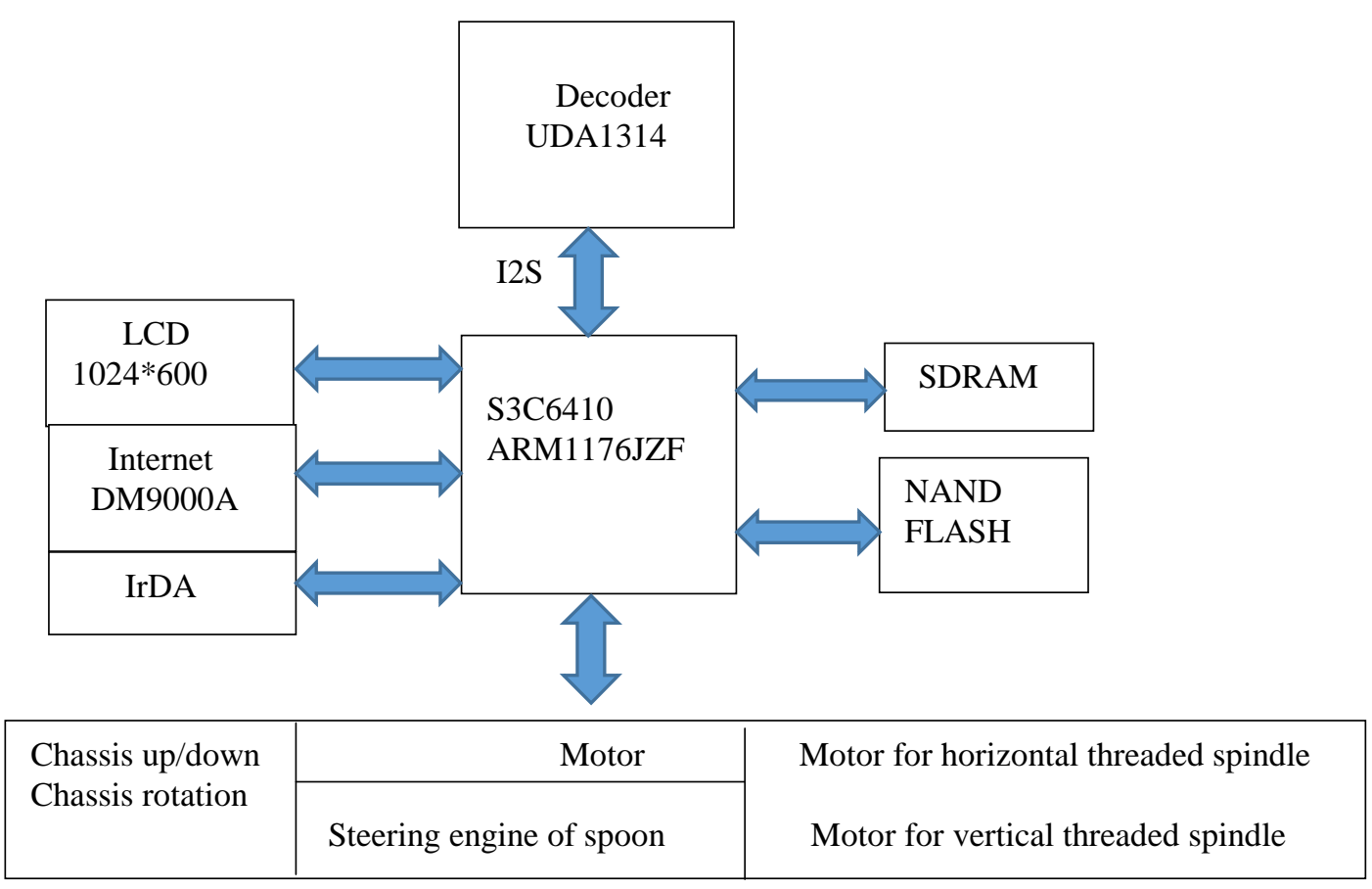

Fig. 1 general design plan

feed users of different heights through its lifting/lowering design. The food change can be realized through its rotation device. Another part is the feeding structure. The upper part of the seat is fixed with the vertical pillar and the chassis in a coaxial mode. The upper end of the vertical pillar is fixed with vertical bracket, vertical threaded spindle slider, horizontal threaded spindle slider and rotating steering gear. The steering gear drives the spoon to rotate, scoop from the bowls and feed the eaters through the linkage with the vertical and horizontal threaded spindles.

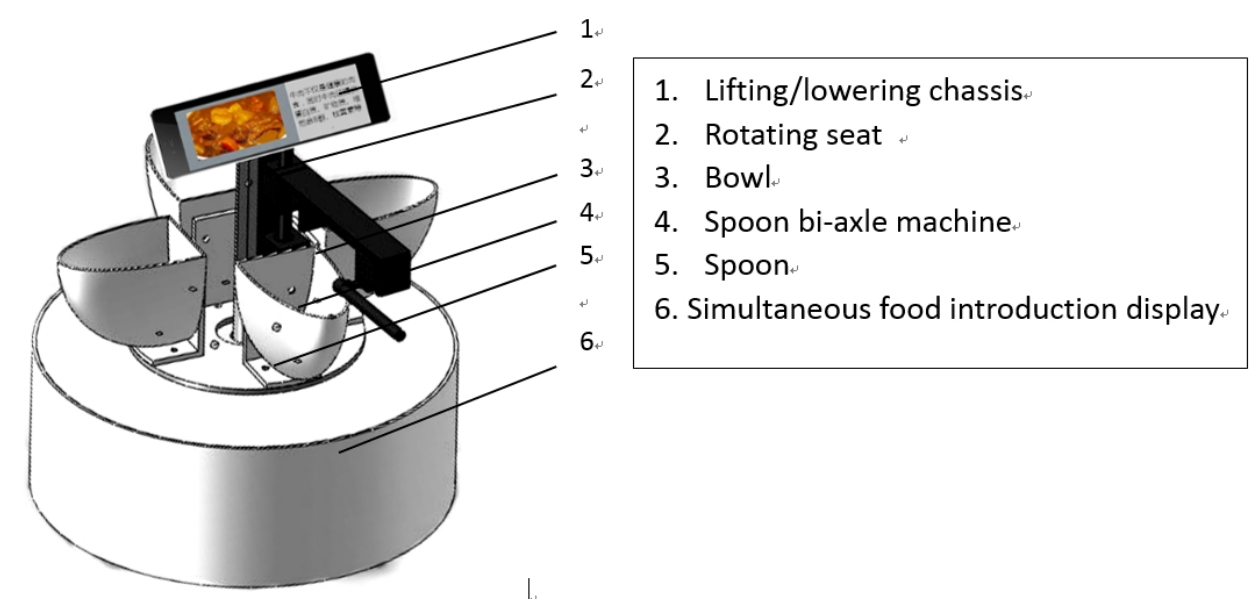

Fig. 2 Structure of desktop meal-assistance machine 


\section{Selection of control modes}

Multiple control modes are needed to help the upper-limb-disabled and senior eaters[10]. To equip the meal-assistance machine with better human-friendliness and usability, two control modes and one interaction mode are applied. Foot switch and voice controller are used for the control.

Voice control Under the voice control mode, users may control the action of meal-assistance robot through voice signals. The voice module applies the 16-bit high-performance SPCE061A singlechip launched by Sunplus as its core. Voice control process mainly consists of two stages. Training is the first stage. By means of training, the system's specification parameters will be obtained, and stored in the template library made up by FLASH memorizers[4]. Voice recognition is the second stage. Audio signals are picked up by a microphone, then encoded to match with the existing digital voice signals in the voice function library in the memorizers for mode recognition through certain audio processing algorithm and corresponding acts and operation. We can store the user's voice, such as "taking food ", "'reset" and "rotating", in the template library as corresponding action operation voice signals. When users give these orders, the system may recognize and execute corresponding actions.

Foot switch control As the seniors often have trembling hands and the upper-limb disabled cannot control with hands, the application of foot switch control is a fairly good solution. With the foot switch, the user can treadle the switches for "taking food", "rotating" and "stop" in turn to control the robot and complete the food-taking action. When pressing the Rotating switch, the chassis will rotate to present the bowl with food. When the eater wants the food, he/she can treadle the Taking switch to order the robot to move the spoon and scoop the food. When the eater wants the robot to be at the initial status, he/she can treadle the Reset switch to achieve that.

Information display module In order to offer good meal experience, the meal-assistance machine is equipped with an information display module which has a wireless receiver and can receive and display the text and voice information on the meal. The information display module will also give a prompt when receiving orders for any switches of system status. For example, when treadling the switch for changing food, the information screen will display "Changing Food" and the food to be taken in the next spoon. When recognizing the eater's voice, for instance, "Meal Completed", the system will reset and be locked with a prompt "Meal Completed" on the screen.

\section{Conclusion}

The paper introduces a desktop meal-assistance robot to help the dining of some senior or upper-limb-disabled persons which is easy to control and tailor-made for Chinese dining habit. Its simple and reliable chassis enables the change of food tray's height and position. The feeding action is performed through the steering gear and threaded spindle slider. The interactions among foot-control switches, voice control and information display module improves the user friendliness. The prototype test indicates that the meal-assistance robot can successfully realize food taking and help users to dine by himself/herself. The meal-assistance robot features simple structure, high safety and reliability, easy operation and mass production.

\section{Acknowledgements}

This work was financially supported by the Beijing Polytechnic College Scientific research project (bgzykyz201403)\& Beijing Young Talents Project- research on Meal-assistance Robot (2014-2016)

\section{References}

1. Li Yantao; Study on Prototype R\&D and Control of Meal-assistance Robot, [D].

2. Zhao Can, Lin Hua, Meng Cu, Wang Tianmiao, Liu Sen, Yao Yuan; Design and Application of ARM-based Multiple-axle Servo Controller, 2006(4):31 
3. Dong Leigang, Cui Xiaowei, Zhang Dan, Zhang Hua, Design of ARM+LINUX-based Video Monitoring System[J]. Computer CD Software and Applications, 2014(03)

4. Xu Zihao, Zhang Tengfei, Smart Housing System Design Based on Voice Recognition and Infinite Sensing Network, Computer Measurement \& Control, 2012.20(1) 